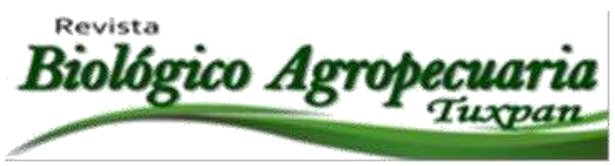

\title{
Alternativas para el control de Diaphorina citri (Kuwayama) con insecticidas botánicos, en Citrus latifolia tanaca, Tlapacoyan, Veracruz.
}

\author{
Alternatives to control Diaphorina citri (Kuwayama) with botanical insecticides in Citrus latifolia tanaca, \\ in Tlapacoyan, Veracruz. \\ González Acosta Alfredo ${ }^{1}$, González Castro Alfredo ${ }^{1}$, Mateos Rocha Roberto A. ${ }^{1}$, González Cárdenas Julio C. \\ 1, López Meza Miguel ${ }^{2}$ \\ ${ }^{1}$ Facultad de Ciencias Biológicas y Agropecuarias Campus Tuxpan, México, Universidad \\ Veracruzana. ${ }^{2}$ Facultad de Agronomía, Universidad Autónoma de Sinaloa. \\ ${ }^{凶}$ Autor para correspondencia: alfgonzalez@uv.mx
}

Recibido: 11/01/2016

Aceptado: 06/06/2016

\section{RESUMEN}

Para el manejo Diaphorina citri(Kuwayama), se evaluaron, en lima persa (Citrus Latifolia Tan.), en la región de Tlapacoyan, Ver., México. Otoño-Invierno, 2008-2009, diferentes, productos con insecticidas botánicos. BIO-Die PROGRANIC ${ }^{\circledR}$ PROGRANIC ${ }^{\circledR}$ Omega, PROGRANIC ${ }^{\circledR}$ Gama, PROGRANIC $^{\circledR}$ UltraluxS. PROGRANIC ${ }^{\circledR}$ Oil Aceite y Testigo. Se utilizó un diseño de bloques completos al azar con 4 repeticiones, evaluando las poblaciones de adultos, ninfas, a través de análisis de varianza mediante la prueba de comparación de medias. Se realizó un conteo previo para establecer el umbral de aplicación con la sola presencia de adultos o ninfas por cada brote recién emergido, para determinar la persistencia de los productos durante periodos de 7, 17, 27 y 37 días, en cada unidad experimental se realizo aplicaciones y evaluaciones. Los efectos más marcados sobre la población de ninfas se manifestaron en las variantes con Oíl, Omega, Biodie, Gama, NeemAcar, Ultralux-s que no difirieron estadísticamente entre ellos y por último difiriendo de todos los demás el testigo. En adultos Diaphorina citri, todos los tratamientos con extractos y aceites difirieron estadísticamente del testigo, después del testigo, a nivel poblacional, se ubicó el extracto Gama, que no marco diferencia del aceite OIL y este a la vez de el extracto vegetal Ultralux-s, NeemAcar y Biodie y por último el Omega que difirió de todas las demás variantes. Estos productos pueden utilizarse exitosamente como alternativa en el manejo integrado de esta plaga.

Palabras clave: Diaphorina citri, insecticidas botánicos, Citrus latifolia tanaca 


\section{ABSTRACT}

Different botanical products to control Diaphorina citri (Kuwayama) were evaluated in Persian lime (Citrus Latifolia Tan.), in Tlapacoyan, Ver., México during the cycle autum-winter 2008-2009. Botanical products included BIO-Die ${ }^{\circledR}$. PROGRANIC ${ }^{\circledR}$ PROGRANIC ${ }^{\circledR}$ Omega, PROGRANIC ${ }^{\circledR}$ Gama,PROGRANIC ${ }^{\circledR}$ UltraluxS. PROGRANIC ${ }^{\circledR}$ Oil. A randomized complete block design with 4 repetitions was used. Data on nymph and adult populations were collected and analyzed by using ANOVA and differences between treatments were analyzed by mean comparisons. A previous determination of the number of adults or nymphs in each recently emerged shoot was conducted to establish a threshold level of application. Persistence of the products during periods of 7, 17, 27 and 37 days, in each experimental unit sprays and evaluations were conducted. Significant effects were observed on nymph populations with the treatments Oíl, Omega, Biodie, Gama, NeemAcar, Ultralux-s; treatments that did not showed statistical differences among them, but statistical difference was observed between them and the control treatment. In Diaphorina citri adult population, all treatments were statistically different in comparison with the control treatment. An increased effect on adult populations was observed on Gama and OIL, then plant extract Ultralux-s, NeemAcar and Biodie, followed by Omega, treatment that was statistically different in comparison with all treatments. These products can be successfully used as alternatives in the integrated management of this pest.

Keywords: Diaphorina citri, botanical insecticides, Citrus latifolia tanaca

\section{INTRODUCCIÓN}

En México se cultivan más de 500 mil hectáreas de cítricos, donde aproximadamente el $69 \%$ corresponden a naranjas, $21 \%$ a limón mexicano, $5 \%$ a lima persa y el resto a mandarinas, toronjas, pomelos, entre otros. (Sánchez y Anguiano et al., 2004). En la temporada 2005-2006 se produjeron en el mundo 72.8 millones de toneladas de cítricos. De ellas el 47.1 correspondieron a naranjas, 16.0 a tangerinas, 4.3 a limón, 2.2 a toronjas, 3.2 a otras especies. De la superficie citrícola mundial se consume el $68 \%$ en fresco y el $32 \%$ se procesa. Dentro del grupo de las frutas cítricas que México exporta destacan los limones y las limas, especialmente la lima persa, producto que totalizó ventas al exterior por 193 millones de dólares en el 2005 que representó un aumento del $3.2 \%$ con respecto al año 2004, las toronjas 5.6 millones de dólares, naranjas 3.4 millones de dólares y mandarinas 1.7 millones de dólares (FAO, 2006).
En Veracruz, principal productor de cítricos en México, se tienen registradas 200,467 hectáreas sembradas, de las cuales 148,523 son de naranja, 24,794 de lima persa, 6,731 de toronja y 20,419 corresponden a otras especies. La producción es de 1'758,591 toneladas de naranja, 332,343 toneladas para lima persa y para toronjas es de 229,894 toneladas, los promedios de cosecha andan de la siguiente manera 11.8 toneladas para naranja, 13.4 para lima persa y 34.2 para toronjas. Los cítricos son afectados por diferentes organismos nocivos como lo es el psílido asiático de los cítricos (Diaphorina citri Kuwayama) y psílido africano de los cítricos (Trioza erytreae). Siendo transmisores de una enfermedad que se conoce con el nombre de Huanglongbing (HLB) y es una de las pocas enfermedades que es considerada un verdadero factor limitante para la producción de cítricos El vector de esta enfermedad es la Diaphorina citri por lo que es necesario impedir el ingreso de material de

Revista Científica Biológico Agropecuaria Tuxpan 4 (1) ISSN: 2007-6940 
propagación en forma ilegal para prevenir el ingreso de la enfermedad al país. En este sentido se hace indispensable buscar nuevas alternativas para el control de dicho psílido, así como ampliar las posibilidades del uso de los productos orgánicos a fin de evitar el surgimiento de posibles fuentes de resistencia (De la luz, 2006).

Dadas las nuevas regulaciones que restringen la presencia de pesticidas en la fruta, hay una tendencia a favorecer los controles a inicios de la brotación. Las razones por las cuales el control químico de las plagas de los cítricos no ha resultado ser tan efectivo es debido a que los productores: a) No monitorean, b) Escaso control de plagas, c) No realizan una buena cobertura al aplicar los insecticidas (mojar toda la planta), d) Usan Insecticidas inadecuados, e) Usan dosis inadecuadas del ingrediente activo, y f) la escasa información acerca del momento óptimo de control. Los insecticidas generalmente interrumpen las relaciones tróficas de éstas especies benéficas, ocasionando incrementos poblacionales de las plagas, que en algunas situaciones, rebasan los niveles previos al implemento del control. Debido a similitudes fisiológicas básicas entre las plagas y los insectos benéficos, los plaguicidas pueden incurrir en severa mortalidad en ambos grupos de organismos. Esto se da principalmente con insecticidas que afectan al sistema nervioso, que comprenden la mayoría de productos actualmente utilizados Prácticamente en todos los cultivos donde regularmente se usan insecticidas, se desarrolla un desequilibrio de ciertas plagas, resultando otras nuevas plagas y lo cual provoca ataques más severos de las ya existentes (Suárez, 2004).

La agricultura moderna ha depositado una confianza excesiva en los insecticidas sintéticos para el control de plagas. La industria ha desarrollado cientos de químicos para controlar un amplio rango de plagas que incluyen insectos, ácaros, nemátodos, malezas, bacterias, virus y hongos. A pesar de que estos tóxicos están dirigidos al control de plagas en cultivos, muchos de ellos son biocidas de amplio espectro que tienen serios efectos en otros organismos. Aún los insecticidas bioracionales desarrollados, los cuales se basan en productos naturales y que son más específicos, pueden tener efectos de largo alcance. La mortalidad directa o subletal de los insecticidas sobre los enemigos naturales puede ser causada por contacto directo, contacto con residuos o a través de la cadena alimenticia. Sin embargo, el efecto de los insecticidas sobre los enemigos naturales ha sido menos estudiado que el efecto que estos tienen sobre las plagas, ya que estas son el principal objetivo del control (Croft, 1989).

Por ello en el este trabajo de investigación se evaluarán 6 insecticidas botánicos para el control de Diaphorinacitri en árboles de lima persa. Se trazaron los siguientes objetivos:

1. Disminuir las poblaciones de Diaphorinacitri en lima persa ( $C$. Latifolia Tan) con la aplicación de insecticidas botánicos.

2. Comparar la eficacia de los insecticidas botánicos para el control de adultos y ninfas de Diaphorinacitri aplicados en lima persa en el municipio de Tlapacoyan, Ver., México.

\section{MATERIALES Y MÉTODOS}

El presente trabajo se realizó en la finca propiedad del señor Pedro González Huerta en

el Ejido Arroyo Piedra, Municipio de Tlapacoyan, Veracruz, el material vegetal que se utilizo fue plantas adultas de lima persa (Citrus Latifolia) con una edad promedio de 5 años, injertadas en patrón de naranjo agrio (Citrus aurantium) establecidos en un marco real de plantación (7X7). Se seleccionaran árboles que presenten características uniformes, que sean todos de un mismo porte o altura, que presenten brotes con presencia de diaphorina, que se encuentren en buen estado nutrimental y que todos estén bajo una misma sección. 
El diseño experimental fue el de bloques completos al azar. La unidad experimental fue el dosel completo de cada árbol. Se aplicaron 6 árboles por cada tratamiento y se incluyeron 4 repeticiones, dando un total de 24 árboles por tratamiento (168 árboles totales). La parcela útil para cada tratamiento fue el dosel medio de los 6 árboles en donde se etiquetaron 4 brotes/árbol ( 1 en cada punto cardinal), dando un total de 24 brotes por unidad experimental. Los tratamientos se listan en el cuadro 1.

\begin{tabular}{|c|c|c|}
\hline Tratamientos & Ingrediente Activo & L/ha \\
\hline 1. BIO-Die ${ }^{\circledR}$ & Argemonina, berberina, ricinina y $\alpha$-Terthienyl al $12.0 \%$. & 1.0 \\
\hline $\begin{array}{l}\text { 2. PROGRANIC } \\
\text { NeemAcar }\end{array}$ & $\begin{array}{c}55.0 \% \text { de extracto de neem(Azadirachta indica) y } 15.0 \% \\
\text { de extracto de canela (Cinnamomumzeylandicum) }\end{array}$ & 1.0 \\
\hline $\begin{array}{l}\text { 3. PROGRANIC } \\
\text { Omega }\end{array}$ & Extracto de chicalote (Argemone mexicana) al $90.0 \%$ & 1.0 \\
\hline 4. PROGRANIC ${ }^{(B)}$ Gama & $\begin{array}{c}\text { Extracto de ajo (Allium cepa), chile picante } \\
\text { (Capsicumfrutescens) y canela } \\
\text { (Cinnamomumzeylandicum) al } 60.0 \%\end{array}$ & 1.0 \\
\hline $\begin{array}{l}\text { 5. PROGRANIC } \\
\text { Ultralux S }\end{array}$ & Sales potásicas de ácidos grasos al 50.0\%. & 1.0 \\
\hline 6. PROGRANIC ${ }^{(B)}$ Oil & $\begin{array}{c}\text { Aceite de soya (Glycinemax), cártamo } \\
\text { (Carthamustinctorius) y girasol (Helianthusannus) al } \\
95.0 \%\end{array}$ & $\begin{array}{l}1.5 \mathrm{~L} / 100 \mathrm{~L} \mathrm{de} \\
\text { agua }\end{array}$ \\
\hline 7. Testigo Absoluto. & Agua & Calibración \\
\hline
\end{tabular}

Cuadro 1. Tratamientos incluidos para el control de Diaphorina en lima persa (Citrus Latifolia Tan.), en la región de Tlapacoyan, Ver., México. Otoño-Invierno, 2008-2009.

La dosis de aplicación de los productos se indica en el cuadro 1. Se realizó un conteo previo para establecer el umbral de aplicación con la sola presencia de adultos o ninfas por cada brote recién emergido. A fin de determinar la persistencia de los productos durante periodos de 7, 17, 27 y 37 días, en cada unidad experimental se realizo aplicaciones y evaluaciones.

Las aplicaciones se realizaron durante las primeras horas de la mañana a fin de evitar el calor intenso y afectación a los productos. El método de aplicación fue por aspersión foliar utilizando una bomba de aspersión manual que se calibro para un gasto de 200 L/ha. Se usó Prolux ${ }^{\circledR}$ Ultra como coadyuvante (surfactanteextensor-penetrante). El agua para la aplicación se acondiciono a un $\mathrm{pH}$ en el rango de 6.5 a 7.0 .

\section{Tamaño de muestra, tipo y frecuencia de muestreo.}

La cuantificación de adultos y ninfas de Diaphorina fue visual utilizando una lupa de $5 \mathrm{X}$ en caso necesario se muestreo los 4 brotes/árbol en su totalidad. Los datos se registraron en cada fecha de muestreo. A fin de determinar la persistencia del producto, los muestreos se realizaron a los 7, 17, 27 y 37 días después de la aplicación (Cuadro 2), y cuando coincida con la aplicación, se realizaron previos a la aspersión.

\section{Revista Científica Biológico Agropecuaria Tuxpan 4 (1)}




\section{Análisis de los datos.}

Se realizó un análisis de varianza y comparación de medias mediante la prueba de Tukey $(\propto=0.05)$, haciendo uso del programa SAS, versión 6.12 (Ray, 1982).

\section{Resultados y discusiones}

Al evaluarse el efecto de los extractos botánicos sobre las ninfas, de Diaphorinacitri el análisis de varianza de clasificación doble realizado con el promedio de los datos de las cuatro evaluaciones arrojó diferencias altamente significativas entre los tratamientos.

Como puede apreciarse en la Fig. 1, todos los tratamientos difirieron estadísticamente del testigo, provocando reducciones de la población de ninfas.

Los efectos más marcados sobre la población de ninfas se manifestaron en las variantes con Oíl, Omega, Biodie, Gama, NeemAcar, Ultralux-s que no difirieron estadísticamente entre ellos y por último difiriendo de todos los demás el testigo.

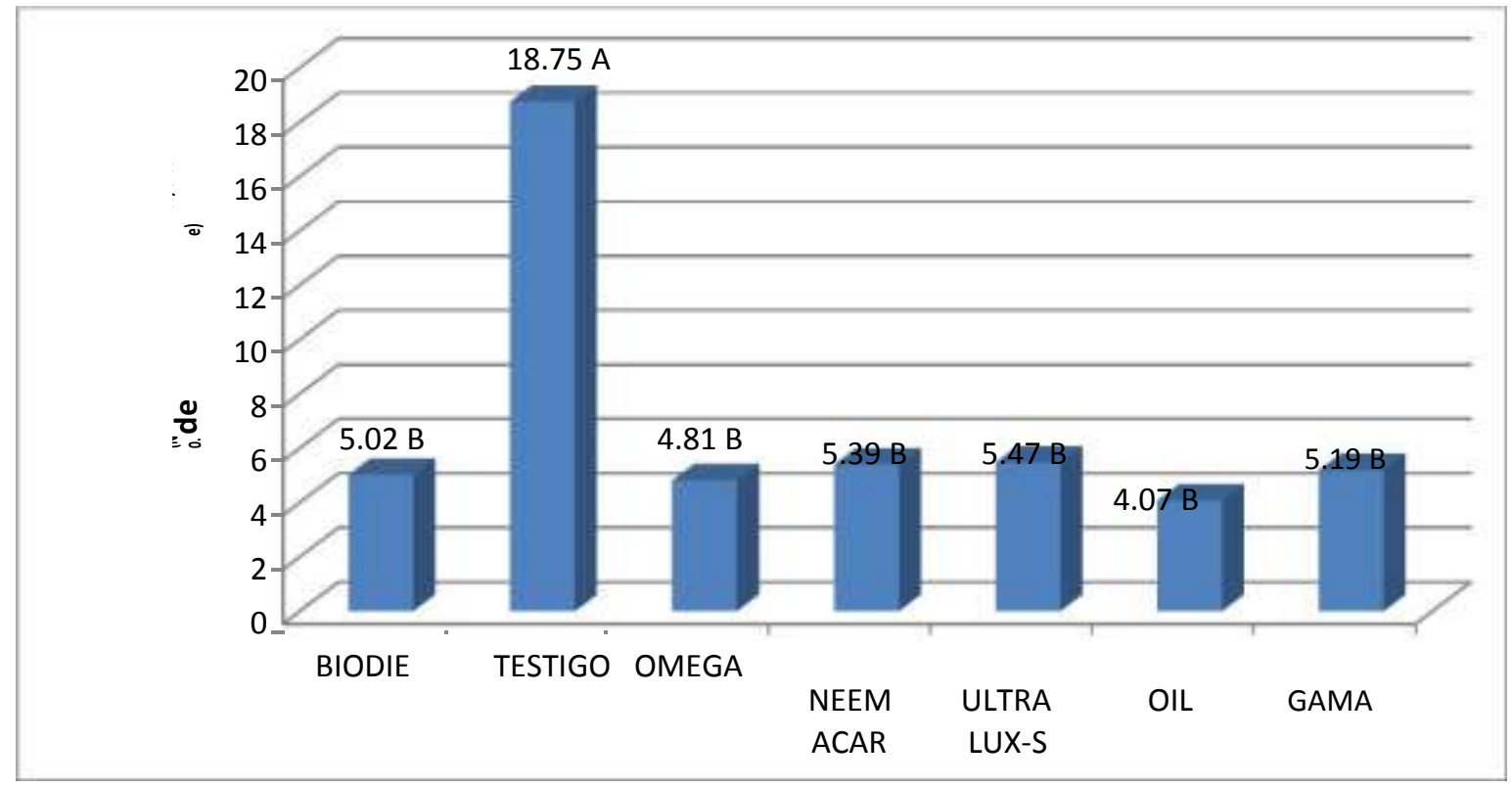

Figura1. Efecto de extractos botánicos sobre ninfas de Diaphorinacitri.

En las Fig. 2 puede observarse el comportamiento de la población de ninfas a través del período en que se realizaron las evaluaciones, a partir del momento en que comenzaron las aplicaciones, la población de ninfas bajó en todos los casos, manteniéndose posteriormente fluctuando en niveles claramente diferentes al testigo. 


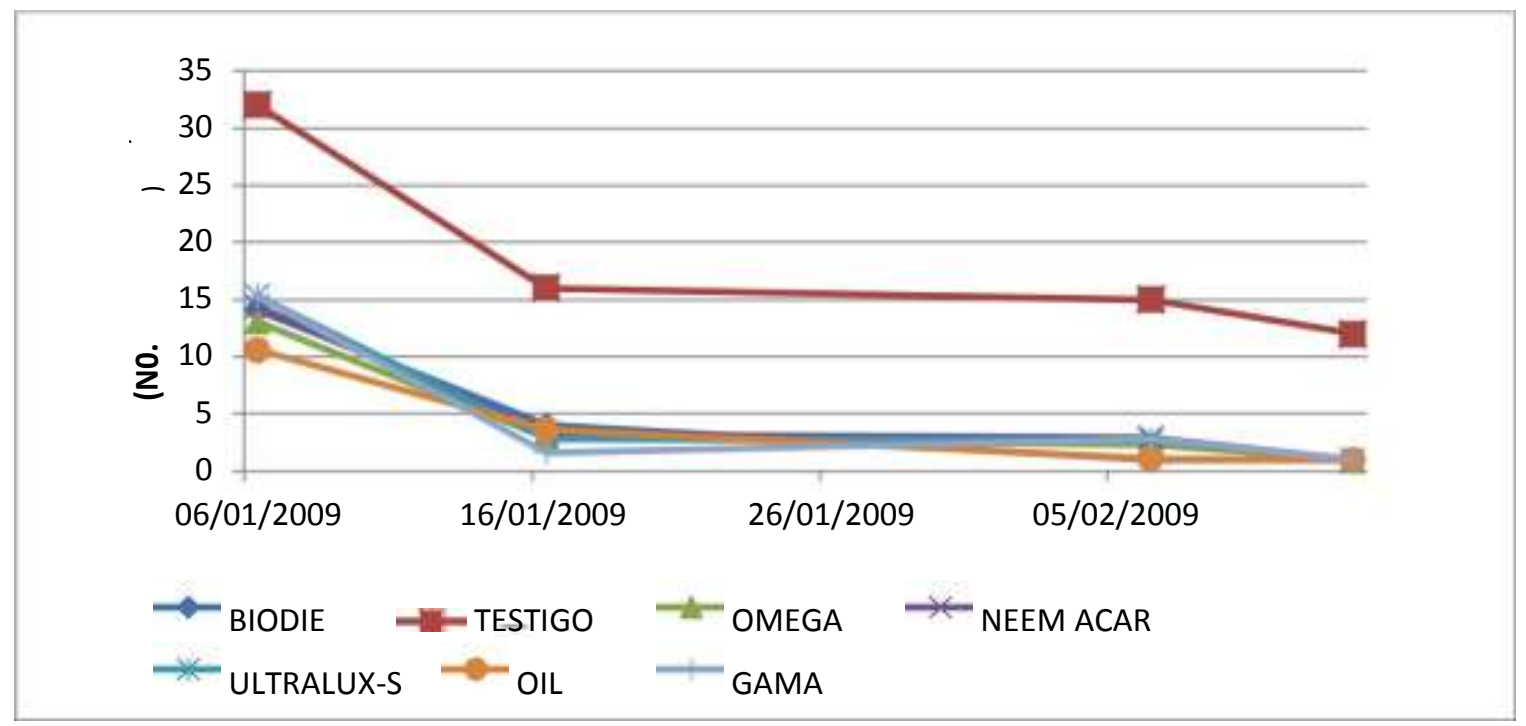

Figura 2. Efectos de extractos botánicos sobre las poblaciones de ninfas de Diaphorinacitri.

Cuando se aplicó un análisis de clasificación doble al promedio de las cuatro evaluaciones realizadas sobre la población de adultos Diaphorina citri, se manifestaron diferencias altamente significativas entre los tratamientos. Los resultados del ensayo se muestran en la Fig.3. En la misma se puede observar que todos los tratamientos con extractos y aceites difirieron estadísticamente del testigo. A continuación del testigo, en cuanto a nivel poblacional, se ubicó el extracto Gama, que no marco diferencia del aceite OIL y este a la ves del extracto vegetal Ultralux-s, NeemAcar y Biodie y por último el Omega que difirió de todas las demás variantes.

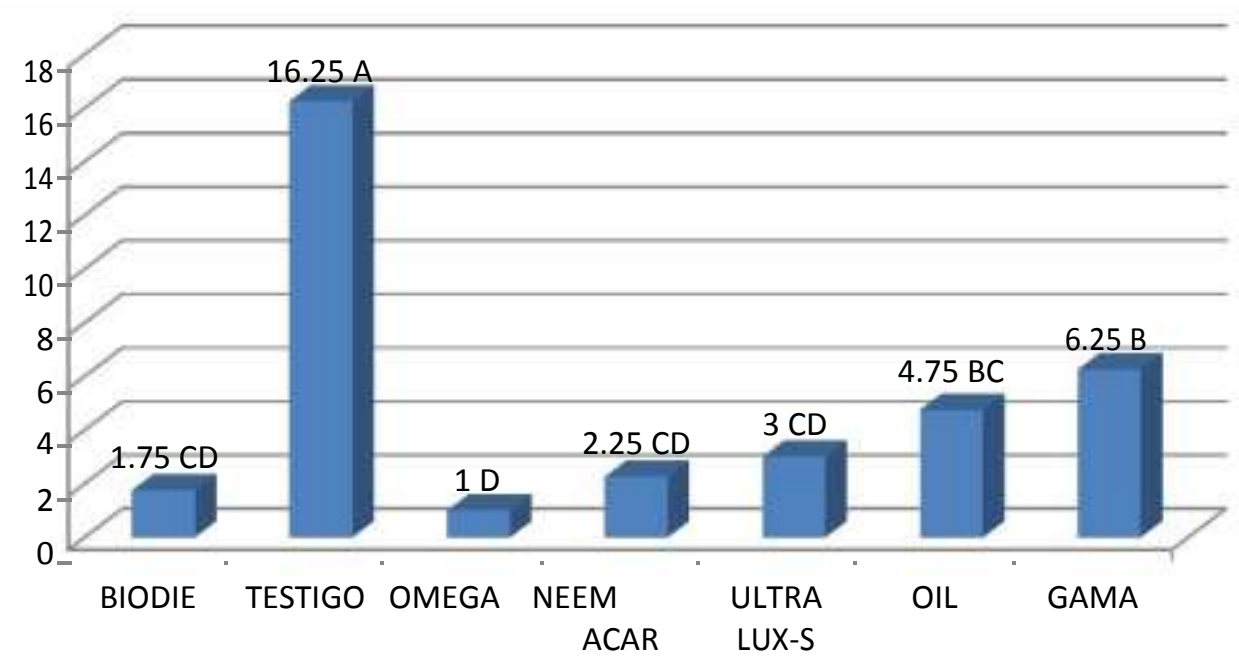

Figura 3. Efectos de extractos botánicos sobre adultos de Diaphorinacitri. 
En la Fig.4 se observa que los diferentes tratamientos con extractos y aceites durante el periodo de las cuatro evaluaciones fueron capaces de mantener las poblaciones de adultos de Diaphorinacitri en niveles mucho más bajos que el testigo, durante el tiempo que duró el ensayo.

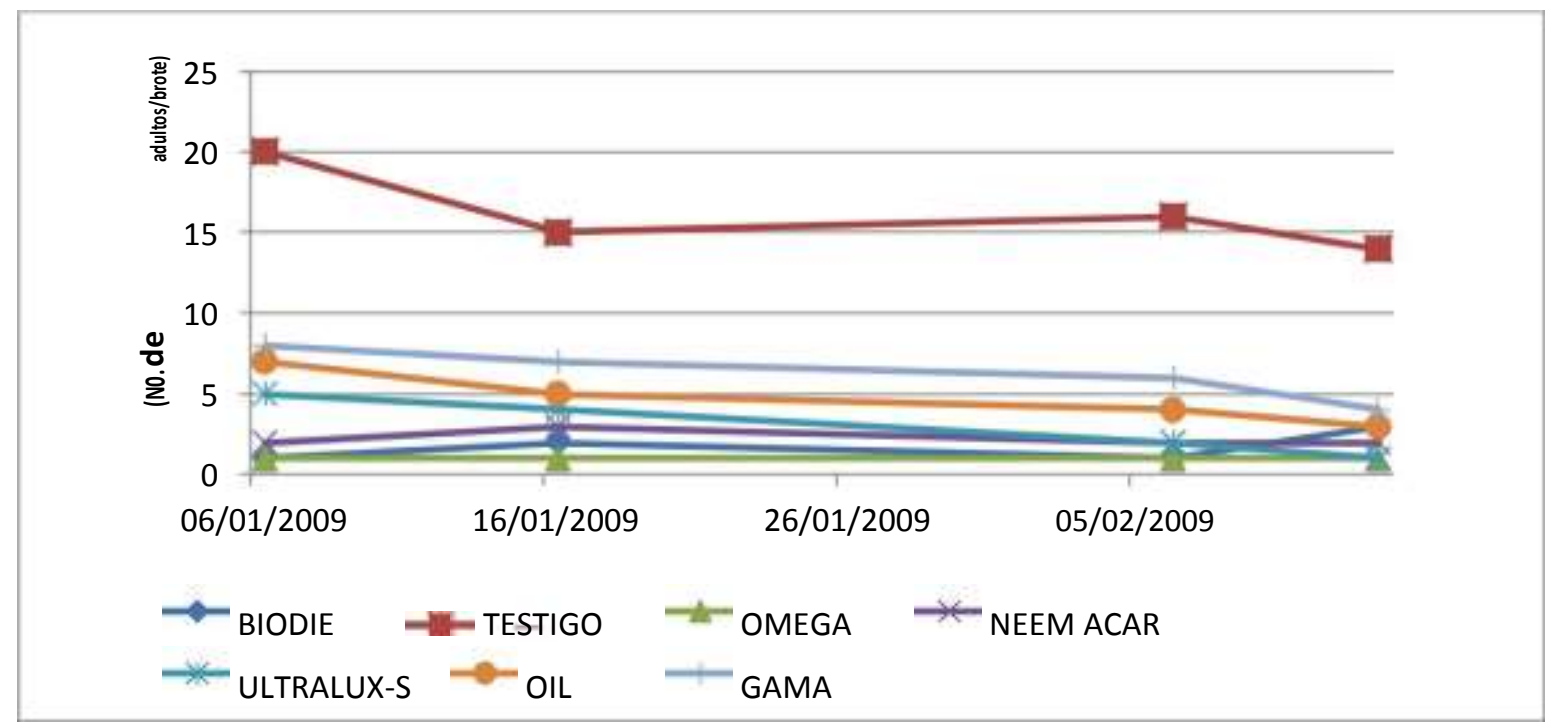

Figura 4. Efectos de extractos botánicos sobre las poblaciones de adultos de Diaphorinacitri.

Los resultados aquí mostrados concuerdan con lo informado por Silva et al. (2002), Valderrábano y Uriarte (2001) y Aguiar et al. (2003), quienes trabajaron con extractos de plantas y encontraron efecto antialimentario y ovicida en varios de ellos, otros autores Curti y Díaz (1993) estudio la efectividad biológica de tres insecticidas botánicos, para controlar plagas en lima persa, evaluando nueve tratamientos que fueron Bio-die ${ }^{\circledR}$ : 1) 1.0, 2) 1.5 , 3) 2.0; Progranic ${ }^{\circledR}$ Cinnacar: 4) 1.0, 5) 2.0, 6) 3.0; Progranic ${ }^{\circledR}$ Nimicide 80: 7) 1.0, 8) 2.0 y 9) 3.0 L/ha ${ }^{-1}$, comparado con los testigos químico, 10) Perfekthion $0.5 \mathrm{~L}_{\mathrm{ha}}{ }^{-1}$ y 11) Absoluto. Los insecticidas botánicos a las diferentes dosis evaluadas no causafitotoxicidad a hojas, flores $\mathrm{y}$ frutos de lima persa. Este estudio de efectividad biológica indica que el BIO-Die ${ }^{\circledR}$, Progranic ${ }^{\circledR}$ CinnAcar y Progranic ${ }^{\circledR}$ Nimicide 80, controlan diaphorina, pulgones, ácaro blanco y piojo harinoso, por lo que se deben de implementar en el programa de manejo integrado fitosanitario delima persa, con las restricciones mencionadas para cada insecticida botánico. Otros investigadores, Weathersbee y Mackenzie (2005), observaron los efectos biológicos de la azadiractina contra Diaphorinacitri, resultando ser efectivo sobre ninfas con una mortalidad de 74 a $92 \%$ después de siete días de aplicación de este insecticida botánico. Además, ellos mismos observaron que el producto no causó ninguna fitotoxicidad en el follaje o la fruta de los cítricos.

\section{CONCLUSIONES}

Para las condiciones de la región de Tlapacoyan, Veracruz, México, las alternativas para el manejo de Diaphorinacitri en el cultivo de lima persa, basadas en dos aplicaciones semanales de extractos botánicos, lograron reducir las poblaciones del fitófago en forma sostenida, se mantuvieron las poblaciones de ninfas alrededor de 4.7 por brotes, mientras que en el testigo se llegó a evaluar un promedio de 18 ninfas por brotes, El control de adultos fue 
mayor donde se realizaron los tratamiento con BIODIE, OMEGA, NEEM ACAR y ULTRA LUX-S, llegando a un rango de uno a tres adultos por brotes, no ocurriendo así en los demás tratamientos, donde el número de adultos por brotes alcanzó una cifra de 4.74 a 6.25 , y a la vez todos los tratamientos fueron superados por el testigo, donde se alcanzó un total de 16.25.

\section{LITERATURA CITADA}

Aguiar, A., 2003.Feeding deterrence of three vegetable extracts on Bemisia tabaci. Adultos. Manejo Integrado de Plagas y Agroecología 68:62-70.

Croft, B., 1989.Arthropod Biological Control Agents and Pesticides. Corvallis, Oregon United States of America. p.723.

Curti-Diaz, S. A., M. Orosco S., U. Díaz Z., X. Loredo-Salazar, R. Rodríguez M.; R. A. Parra Q. y J. A. Sandoval R. 1993. Manual de producción de los citricos en Veracruz

De la Luz, R, M., 2006. Tratados comerciales para la exportación de la fruta. Senasica, sagarpa. Ponencia $\mathrm{x}$ congreso internacional de la citricultura, $\mathrm{Cd}$. Victoria Tamps.

FAO, 2006. Comité de problemas de productos básicos. Boletin informativo. Pags. 1-14.

Ray, A. 1982. SAS user"s guide. Statistics. SAS institute INC.CARI, M.C

Sánchez, A. H., Anguiano P.S., 2004. Programa nacional de certificación de cítricos en México: Antecedentes, resultados y perspectivas..

Silva, A.G., A.T. Lagunes, C.M. Rodríguez y D.L. Rodríguez. 2002. Insecticidas vegetales: una vieja y nueva alternativa para el manejo de plagas. Manejo Integrado de Plagas y Agroecología 66: 4-12.

Suárez, C, J, M., 2004. Evaluación de acaricidas en el control de araña roja p.
Diaphorina citri en Iima persa C. latifolia, en la región de Martínez de la Torre. Ver, tesis Universidad Veracruzana.

Valderrábano, J.; Uriarte, J., 2001. Effect of certain plant extracts on the destruction of eggs and larvae. ITEA 22(1):179-181. https://doi.org/10.1016/S0367-326X(00)0 0281-1

Weathersbee, A.A.; C.L. Mckenzie.2005. Effect of Neem biopesticide on repellency, mortality, oviposition, and development of Diaphorina citri kuwayama (Homoptera: Psilidae). Florida Entomologist $88 \quad$ (4) 401-407. https://doi.org/10.1653/0015-4040(2005)8 8[401:EOANBO]2.0.CO;2 


\section{Copyright (c) 2016 Alfredo G onzález Acosta, Alfredo González Castro, Roberto A Mateos Rocha, \\ Julio C G onzález Cárdenas y Miguel López Meza}

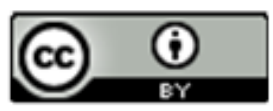

Este tex to está protegido por una licencia licencia Creative Commons 4.0 .

Usted es libre para Compartir — copiar y redistribuir el material en cualquier medio o form ato- y Adaptar el documento — remezclar, transformar y crear a partir del material- para cualquier propósito, incluso para fines comerciales, siempre que cumpla la condición de:

Atribución: Usted debe dar crédito a la obra original de manera adecuada, proporcionar un enlace a la licencia, e in dicar si se han realizado cambios. Puede hacerlo en cualquier form a razonable, pero no de form a tal que sugiera que tiene el apoyo del licenciante o lo recibe por el uso que hace de la obra.

$\underline{\text { Resumendelicencia }}-\underline{\text { Textocompletodelalicencia }}$ 